\title{
A Theorem of Woodin on Mouse Sets
}

\author{
John Steel
}

July 14, 2004

\section{Introduction}

It is well known that for any $n<\omega$, the reals in the minimal fully iterable $L[\vec{E}]$-model with $n$ Woodin cardinals are exactly the reals which are $\Delta_{n+2}^{1}$ in a countable ordinal ([8]). In a similar vein, the reals in the minimal fully iterable $L[\vec{E}]$-model with $\omega$ Woodin cardinals are exactly those which are $\Delta_{1}^{L(\mathbb{R})}$ in a countable ordinal, or equivalently, $\mathrm{OD}^{L(\mathbb{R})}([7])$. Rudominer $([5])$ has extended these theorems to other pointclasses in $L(\mathbb{R})$.

Definition 1.1 $A$ set $X$ of reals is a mouse set iff $X=\mathbb{R} \cap \mathcal{M}$ for some $\omega_{1}+1$-iterable premouse $\mathcal{M}$.

Definition 1.2 For any pointclass $\Gamma$,

$$
C_{\Gamma}=\{x \mid x \text { is } \Delta \text { in some countable ordinal }\}
$$

We relativise to a real $x$ by letting $C_{\Gamma}(x)=C_{\Gamma(x)}$ be the set of reals which are $\Delta(x)$ in some countable ordinal.

In this language, the theorems cited above state that for $\Gamma=\Sigma_{n+2}^{1}$ or $\Gamma=$ $\Sigma_{1}^{L(\mathbb{R})}, C_{\Gamma}$ is a mouse set. ( What is more, however, they also identify the mouse via large cardinal properties.)

It is natural to conjecture that if $\Gamma$ is any level of the (lightface) Levy hierarchy for $L(\mathbb{R})$, then $C_{\Gamma}$ is a mouse set. ( See [5] for a precise version of this conjecture.) In this note we shall prove the following theorem of Hugh Woodin, which settles the matter in many cases. 
Theorem 1.3 (Woodin) Assume $A D^{L(\mathbb{R})}$, let $\lambda$ be a limit ordinal, and let $\Gamma$ be the pointclass of sets which are definable over $J_{\beta}(\mathbb{R})$ for some $\beta<\lambda$; then $C_{\Gamma}$ is a mouse set.

It is easy to see that if $\Gamma$ is as in 1.3 , then $C_{\Gamma}$ is the set of reals which are $\mathrm{OD}^{J_{\beta}(\mathbb{R})}$ for some $\beta<\lambda$.

Woodin actually proved a somewhat more general theorem. It is slightly easier to state the theorem in the context of full $A D$, so that is what we shall do. Recall that, in this context, $\theta$ is the least ordinal not the surjective image of $\mathbb{R}$, or what is the same, the order type of the Wadge hierarchy.

Definition 1.4 Assume $A D$, and let $\alpha<\theta$; then

1.

$$
\begin{aligned}
P_{\alpha}(\mathbb{R}) & =\{A \subseteq \mathbb{R} \mid A \text { has Wadge ordinal }<\alpha\}, \\
P_{\alpha}\left(V_{\omega+1}\right) & =\left\{A \subseteq V_{\omega+1} \mid A \text { is coded by some } B \in P_{\alpha}(\mathbb{R})\right\} .
\end{aligned}
$$

2. $D f_{\alpha}$ is the pointclass consisting of all subsets of $\mathbb{R}$ definable from no parameters over the structure $\left(P_{\alpha}\left(V_{\omega+1}\right), \in\right)$.

Theorem 1.5 (Woodin) Assume $A D$, and let $\alpha<\theta$ be such that $P_{\alpha}(\mathbb{R})$ is closed under $\exists^{\mathbb{R}}$, and every set in $P_{\alpha}(\mathbb{R})$ has a scale in $P_{\alpha}(\mathbb{R})$. Suppose that every countable premouse having an $\omega_{1}$-iteration strategy in $P_{\alpha}(\mathbb{R})$ is tame. Let

$$
\Gamma=\bigcup_{\beta<\alpha} D f_{\beta} ;
$$

then $C_{\Gamma}$ is a mouse set.

An $\omega_{1}$ iteration strategy for a countable premouse can be naturally coded as a set of reals, so the hypothesis of 1.5 makes sense. A premouse is tame if, roughly speaking, it has no extenders overlapping Woodin cardinals.( See [7].) It is worth noting that $A D$ implies $\omega_{1}$ is measurable, and hence that every $\omega_{1}$ iteration strategy on a countable premouse extends to an $\omega_{1}+1$ iteration strategy. $A D$ therefore implies that any two $\omega_{1}$-iterable countable premice have a successful coiteration.

The proof of 1.5 we give here was, at least in its essentials, communicated to the author by Hugh Woodin in January,1994. The author's memory is less than perfect, so there may be some divergence in detail between the proof to follow and Woodin's. This paper is an update of an earlier version in which we proved only 1.3. All unattributed results to follow are due to Woodin. 


\section{Inner model operators}

It has been known for a while, at least implicitly, that 1.5 is true on a cone. In order to explain why, we need the notion of an inner model operator, for which we refer the reader to [6]. Roughly speaking, an inner model operator is a Turing invariant function $M$ associating to each real $x$ a countable set $M(x)$ of reals closed under Turing jump, join, and "recursive in" and such that $x \in M(x)$. Turing invariance means that $x \equiv_{T} y \rightarrow M(x)=M(y)$. (There must also be an accompanying function assigning a wellorder $<_{x}$ to $M(x)$ and such that if $x \equiv_{T} y$, then $M(x)$ can "translate" $<_{x}$ to $<_{y}$.) If $M$ and $N$ are inner model operators, then $M \leq_{m} N$ iff for a cone of $x$, $M(x) \subseteq N(x) . A D$ implies that $\leq_{m}$ prewellorders the inner model operators. (Cf. [6].)

Definition 2.1 An inner model operator $M$ is fine structural iff for a cone of reals $x$, there is an $\omega_{1}+1$-iterable, countable $x$-mouse $\mathcal{P}_{x}$ such that $M(x)=\mathbb{R} \cap \mathcal{P}_{x}$.

(By an $x$-premouse, we mean a level of an $L[\vec{E}]$ model built over $x$; that is, $x$ is thrown in at the bottom.)

Theorem 2.2 (Rudominer, Steel) Assume AD, and let $N$ be a fine structural inner model operator; then for any $M \leq_{m} N, M$ is fine structural.

Theorem 2.2 is proved in $[6$, section 2$]$ in the case that $N$ is the inner model operator $x \mapsto \mathbb{R} \cap L[x]$. The proof given there generalizes routinely so as to yield 2.2.

If $\Gamma$ is as in the hypothesis of 1.5 , then $M$ is an inner model operator, where $M(x)=C_{\Gamma}(x)$ for all $x$. It is a theorem of Woodin that, assuming $A D$, there is a fine structural $N$ such that $M \leq_{m} N$ (see 4.1 below). (One can show more directly that if there are $\omega$ Woodin cardinals with a measurable above, and $\Gamma$ is in $L(\mathbb{R})$, then there is a fine structural $N$ such that $M \leq_{m} N$.) From 2.2 we get that $M$ is fine structural, that is, that $M(x)$ is an " $x$-mouse set" for a cone of $x$. The same argument works for other pointclasses to which Woodin's proof of 1.5 doesn't seem to apply. For example, if $\Gamma$ is a good pointclass ( see definition 3.1 below) in $L(\mathbb{R})$, then $x \mapsto C_{\Gamma}(x)$ is an inner model operator, and if $A D^{L(\mathbb{R})}$ is true, then it is $\leq_{m}$ some fine structural operator in $L(\mathbb{R})$, and hence fine structural itself. On the other hand, there 
are good "lightface" $\Gamma$ in $L(\mathbb{R})$ for which we cannot prove $C_{\Gamma}$ is a mouse set. ( The first such $\Gamma$ is the pointclass $\Sigma_{3}^{J_{2}(\mathbb{R})}$.)

Our questions about mouse sets arose in part from the following conjectures.

Conjecture 1. Assume $A D^{L(\mathbb{R})}$, and let $M$ be an inner model operator in $L(\mathbb{R})$; then for a cone of $x$, there is a wellorder of $M(x)$ in $L(M(x))$.

Conjecture 2 . Let $\mathcal{M}$ be a countable, $\omega$-small, $\omega_{1}+1$ iterable premouse; then there is a wellorder of $\mathbb{R} \cap \mathcal{M}$ in $L(\mathbb{R} \cap \mathcal{M})$.

Any proof of conjecture 2 will no doubt relativise to premice built over a real. Since $L(\mathbb{R})$ satisfies " all $\omega_{1}$ iterable premice are $\omega$-small", and all inner model operators in $L(\mathbb{R})$ are fine structural, this means that a proof of conjecture 2 will no doubt also yield conjecture 1 . Despite the progress represented by 1.3 and 1.5 , both conjectures appear to be open.

\section{Coarse structural $\Gamma$-Woodin mice}

Definition 3.1 A pointclass $\Gamma$ is good iff $\Gamma$ is $\omega$-parameterized, closed under recursive substitution, number quantification, and $\exists^{\mathbb{R}}$, and has the scale property.

One reason to focus on good pointclasses in connection with the $C_{\Gamma}$ operation is the following well known theorem of Kechris:

Theorem 3.2 (Kechris,[2]) Assume $A D$, and let $\Gamma$ be good; then $C_{\Gamma}$ is the largest countable $\Gamma$ set of reals, moreover $C_{\Gamma}$ has a $\Delta$-good wellorder.

We shall use $<_{\Gamma, x}$ for the $\Delta(x)$-good wellorder of $C_{\Gamma}(x)$ provided by Kechris' theorem.

It is useful to extend the operator $x \mapsto C_{\Gamma}(x)$ to countable transitive sets. For $a$ countable transitive and $f: \omega \rightarrow a$ a surjection, we let $x_{f}(\langle m, n\rangle)=0$ iff $f(m) \in f(n)$, and call $x_{f}$ a real coding $a$. Clearly, $x_{f}$ determines $f$. For $b \subseteq a$ and $x=x_{f}$, we let $b_{x}(n)=0$ iff $f(n) \in b$, and say that $b_{x}$ codes $b$ relative to $x$. The space of all $f: \omega \rightarrow a$ is homeomorphic to the Baire space, and the set of surjections is comeager, so we can make sense of the phrase "for comeager many $x$ coding $a$ ". 
Definition 3.3 For a countable and transitive,

$$
C_{\Gamma}(a)=\left\{b \subseteq a \mid \text { for all } x \in \mathbf{R} \text { coding } a, b_{x} \in C_{\Gamma}(x)\right\} .
$$

It is a result of Harrington and Kechris ([1]) that if $\Gamma$ is good and $T$ is the tree of a $\Gamma$-scale on a universal $\Gamma$ set, and enough determinacy (e.g. $A D$ ) is true, then $C_{\Gamma}(x)=\mathbf{R} \cap L[T, x]$ for all reals $x$. This characterization extends easily to $C_{\Gamma}(a)$.

Theorem 3.4 (Harrington and Kechris, [1]) Assume AD. Let $\Gamma$ be a good pointclass, and $T$ the tree of a $\Gamma$-scale on a universal $\Gamma$ set. Let a be countable and transitive; then

1. $C_{\Gamma}(a)=\left\{b \subseteq a \mid\right.$ for comeager many $x$ coding $\left.a, b_{x} \in C_{\Gamma}(x)\right\}$,

2. $C_{\Gamma}(a)=P(a) \cap L(a \cup\{T, a\})$.

Proof.(Sketch) For the nontrivial inclusion in (1), fix any real $y$ coding $a$. Note that we can fix $\alpha$ such that for nonmeager many $x$ coding $a, b_{x}$ is the $\alpha^{t h}$ real in $<_{\Gamma, x}$. Using the $\Gamma$-definability of the "nonmeager many" quantifier applied to $\Gamma$ relations, we get that $b_{y}$ is $\Delta(y)$ in any code for $\alpha$, so $b_{y} \in C_{\Gamma}(y)$. For $(2)$, note $\supseteq$ is trivial by [1]. As to $\subseteq$, let $b \in C_{\Gamma}(a)$; then for comeager many $x$ coding $a, b_{x} \in L[T, x]$ by (1) and [1]. We can then find $x_{0}$ and $x_{1}$ pairwise generic over $L[a \cup\{T, a\}]$ such that $b \in\left(L(a \cup\{T, a\})\left[x_{0}\right] \cap L(a \cup\right.$ $\left.\{T, a\})\left[x_{1}\right]\right)$, so $b \in L(a \cup\{T, a\})$.

We note in passing that there may be no wellorder of $a$ in $L(a \cup\{T, a\})$, and hence no wellorder of $C_{\Gamma}(a)$. We can, however, define in $L(a \cup\{T, a\})$ a natural norm on $C_{\Gamma}(a)$ by letting, for $b \in C_{\Gamma}(a), \varphi_{\Gamma, a}(b)$ be the least $\alpha$ such that for nonmeager many $x$ coding $a, b_{x}$ is the $\alpha^{t h}$ real in $<_{\Gamma, x}$. Every proper initial segment of the prewellorder associated to $\varphi$ is the surjective image of $a$ in $L(a \cup\{T, a\})$. Indeed if we define $\pi=\pi_{\Gamma, a}$ by setting $\pi(p, \alpha)=b$ iff $p \in a^{<\omega}$ and for comeager many $f$ extending $p, b_{x_{f}}$ is the $\alpha^{t h}$ real in $<_{\Gamma, x_{f}}$, then for any $\alpha, \pi^{\prime \prime}\left(a^{<\omega} \times \alpha\right)=\{b \mid \varphi(b)<\alpha\}$, and $\pi$ is in $L(a \cup\{T, a\})$ by the definability of forcing. The order type of $\operatorname{ran}(\varphi)$ is the least ordinal, call it $\theta_{\Gamma}(a)$, which is not the surjective image of $a$ in $L(a \cup\{T, a\})$. (If the order type is strictly less than $\theta_{\Gamma, a}$, then $L(a \cup\{T, a\})$ satisfies that there is a map of $a \times a$ onto $P(a)$.)

The closure of $\Gamma$ under the category quantifiers easily yields the following lemma, whose proof we omit. 
Lemma 3.5 Assume $A D$, and let $\Gamma$ be good; then the relations

$$
\begin{aligned}
C(x, y) \Leftrightarrow & x \text { codes a countable transitive set } a \\
& \text { and } y=b_{x} \text { for some } b \in C_{\Gamma}(a), \\
I(x, y, z) \Leftrightarrow & x \text { codes a countable transitive set } a \text { and } \\
& y \in \text { WO codes an } \alpha<\theta_{\Gamma, a} \text { and } \\
& z \text { codes } \pi_{\Gamma, a}\left\lceil\left(a^{<\omega} \times \alpha\right) \text { relative to } x \text { and } y,\right.
\end{aligned}
$$

are in $\Gamma$.

We need a condensation property of the $C_{\Gamma}$ operation. The first easy lemma is an abstract version of the fact that if $M$ is the transitive collapse of a hull of $\mathrm{L}$, then for any $\beta \in M, P(\beta)^{M}$ is a $\leq_{L}$-initial segment of $P(\beta)^{L}$.

Lemma 3.6 Assume $A D$, let $\Gamma$ be good, and let $T$ be a tree such that $p[T]$ is a universal $\Gamma$ set. Let a be countable and transitive, and suppose $N$ is an admissible set such that $T, a \in N$. Then $C_{\Gamma}(a) \subset N$, and in fact $C_{\Gamma}(a)$ is $\Sigma_{1}^{N}(\{T, a\})$. Moreover, if $\pi: M \rightarrow N$ is elementary and $\pi(\langle\bar{T}, \bar{a}\rangle)=$ $(\langle T, a\rangle)$, then $\pi^{-1} " C_{\Gamma}(a)$ is an initial segment of $C_{\Gamma}(\bar{a})$ under its canonical prewellorder.

Proof.(Sketch) Use 3.5 and the definability of the forcing relation for $\operatorname{Col}(\omega, a)$ over $N$ to prove the first assertion. The "moreover" assertion follows from 3.5 and the fact that $p[\bar{T}] \subseteq p[T]$.

What do we need to add to the hypotheses of 3.6 to guarantee that $\pi^{-1 "} C_{\Gamma}(a)=C_{\Gamma}(\bar{a})$ ? This of course does not follow from the hypotheses of 3.6 themselves, as a simple example with $\Gamma=\Sigma_{2}^{1}, N$ an initial segment of $L$, and $a=\omega$ shows. It suffices to add that there is a tree $\mathrm{U}$ in $\operatorname{ran}(\pi)$ which projects to the complement of a universal $\Gamma$ set, and this would be enough for our purposes here, but we prefer to prove a sharper result which is useful elsewhere. (This sharper result is due to Woodin.) As a bit of motivation, consider the following fact about $\Sigma_{2}^{1}$ and $L:$ if $\pi: M \rightarrow N$ is elementary and $\pi(\bar{\alpha})=\alpha$, and if for each $n<\omega$ the type, with parameters $<\alpha$, of the first $n$ $L$-indiscernibles above $\alpha$ is in $\operatorname{ran}(\pi)$, then $P(\bar{\alpha})^{L} \subseteq M$. Now the type of the first $n L$-indiscernibles yields the first $n$ norms of the Martin-Solovay scale on $\Pi_{2}^{1}$, and it turns out that it is these norms which must be in $\operatorname{ran}(\pi)$. 
Lemma 3.7 Assume $A D$, let $\Gamma$ be good, let $T$ be a tree such that $p[T]$ is a universal $\Gamma$ set, and let $a$ be countable and transitive. Let $M$ and $N$ be admissible sets such that $T, a \in N$ and $N \in M$. Let $\left\langle\psi_{n}\right| n\langle\omega\rangle$ be a scale on $\neg p[T]$, and let $\leq_{n}$ be the prewellorder associated to $\psi_{n}$. Suppose that for each $n$ there is a term $\tau_{n} \in M$ such that whenever $G$ is $M$-generic over $\operatorname{Col}(\omega, N)$,

$$
\tau_{n}^{G}=\leq_{n} \cap M[G] .
$$

Let $\pi: \bar{M} \rightarrow M$ be elementary with $\pi(\bar{a})=a$, and suppose in addition $T, N$ are in $\operatorname{ran}(\pi)$, and

$$
\left\{\tau_{n} \mid n<\omega\right\} \subseteq \operatorname{ran}(\pi) .
$$

Then $C_{\Gamma}(\bar{a}) \in \bar{M}$, and in fact

$$
\pi\left(C_{\Gamma}(\bar{a})\right)=C_{\Gamma}(a) .
$$

Proof.Let $Z=\neg p[T]$ be our universal $\breve{\Gamma}$ set. Let $\varphi_{n}$ be a term in $M$ such that for any $G, \varphi_{n}^{G}$ is the norm on $Z \cap M[G]$ determined by $\tau_{n}^{G}$. Let $\dot{U}_{n}$ be a term for the $n^{\text {th }}$ level of the tree associated to these norms; that is, for any $M$-generic for $\operatorname{Col}(\omega, N)$ set $G$,

$$
\dot{U}_{n}^{G}=\left\{\left(x \mid n,\left(\varphi_{0}^{G}(x) \ldots \varphi_{n-1}^{G}(x)\right)\right) \mid x \in Z \cap M[G]\right\} .
$$

Since $\tau_{n}^{G}=\tau_{n}^{H}$ whenever $M[G]=M[H]$, we see that $\dot{U}_{n}{ }^{G}$ is independent of $G$ and in the ground model; that is, there are $U_{n} \in M$ such that

$$
\dot{U}_{n}{ }^{G}=U_{n} \text {, for all } M \text {-generic over } \operatorname{Col}(\omega, N) \text { sets } G \text {. }
$$

Let $U$ be the tree whose $n^{\text {th }}$ level is $U_{n}$. (Of course, $U \notin M$ is possible.)

Claim. For any $M$-generic over $\operatorname{Col}(\omega, N$ set $G, Z \cap M[G] \subseteq p[U]$. Moreover, $p[U] \subseteq Z$.

Proof. The first statement is obvious. Now suppose $(x, f) \in[U]$. Pick any $G$ which is $M$-generic over $\operatorname{Col}(\omega, N)$. Since the $n^{\text {th }}$ level of $U$ is $\dot{U}_{n}{ }^{G}$, for all $n$, we can find for each $n$ a real $x_{n} \in Z \cap M[G]$ such that $x\left|n=x_{n}\right| n$ and $\forall i<n\left(\varphi_{i}^{G}\left(x_{n}\right)=f(i)\right)$. But then for each $i, \varphi_{i}^{G}\left(x_{n}\right)$ is eventually constant as $n \rightarrow \omega$, and hence $\psi_{i}\left(x_{n}\right)$ is eventually constant as $n \rightarrow \omega$. Thus $x \in Z$. 
The main reason that the $C_{\Gamma}$ completeness of $M$ reflects to $\bar{M}$ is that it is a $\breve{\Gamma}$ statement. More precisely, we can fix a $k_{0}<\omega$ such that for all reals $x$ and $y$,

$$
\left(k_{0}, x, y\right) \in Z \leftrightarrow\left(\forall w \in C_{\Gamma}(x)\right)(\exists i \in \omega)\left(w=(y)_{i}\right) .
$$

Let us fix terms $\sigma$ and $\rho$ such that for any $M$-generic over $\operatorname{Col}(\omega, N)$ set G, $\sigma^{G}$ is the real coding $a$ and $\rho^{G}$ the real enumerating $\mathbf{R} \cap N\left[\sigma^{G}\right]$ determined by $G$. We have then that $\emptyset$ forces over $M$ in $\operatorname{Col}(\omega, N)$ the sentences " $\left(\check{k_{0}}, \sigma, \rho\right) \in$ $\bigcap_{i<n} \operatorname{dom}\left(\varphi_{i}\right) "$ and $"\left(\left(\check{k_{0}}, \sigma, \rho\right) \uparrow n,\left\langle\varphi_{0}\left(\left(\check{k_{0}}, \sigma, \rho\right)\right), \ldots, \varphi_{n-1}\left(\left(\check{k_{0}}, \sigma, \rho\right)\right)\right\rangle\right) \in \check{U}_{n}$. Letting $\left(N, \sigma, \rho, \varphi_{i}, U_{i}\right)=\pi\left(\bar{N}, \bar{\sigma}, \bar{\rho}, \bar{\varphi}_{i}, \bar{U}_{i}\right)$, we have the same sentences with bars everywhere are forced by $\emptyset$ over $\bar{M}$ in $\operatorname{Col}(\omega, \bar{N})$.

This means that if $H$ is $\bar{M}$-generic over $\operatorname{Col}(\omega, \bar{N})$, then $\left(k_{0}, \bar{\sigma}^{H}, \bar{\rho}^{H}\right) \in$ $p[U]$, the witness being $\left\langle\pi\left(\bar{\varphi}_{i}^{H}\left(\left(k_{0}, \bar{\sigma}^{H}, \bar{\rho}^{H}\right)\right)|i<\omega\rangle\right.\right.$, and therefore $C_{\Gamma}\left(\bar{\sigma}^{H}\right) \subseteq$ $\bar{M}[H]$. Since this is true for all such $H, C_{\Gamma}(\bar{a}) \subseteq \bar{M}$. We leave the proof that $\pi\left(C_{\Gamma}(\bar{a})=C_{\Gamma}(a)\right.$ to the reader.

There is a stronger version of 3.7 proved in [9].

We only need here a crude version of 3.7, and as it has a cleaner statement, we record it now.

Corollary 3.8 Assume $A D$, let $\Gamma$ be good, and let $T$ and $U$ be trees projecting to a universal $\Gamma$ set and its complement. Let a be countable and transitive, and let $M$ be a $\Sigma_{2}$-admissible set such that $T, U, a \in M$. Let $\pi: \bar{M} \rightarrow M$ be elementary, with $\pi(\bar{a})=a$ and $T, U \in \operatorname{ran}(\pi)$; then $C_{\Gamma}(\bar{a}) \in \bar{M}$, and $\pi\left(C_{\Gamma}(\bar{a})\right)=C_{\Gamma}(a)$.

We turn now to $\Gamma$-Woodin mice.

Definition 3.9 Let a be countable and transitive; then $a$ is $\Gamma$-amenable iff whenever $x \in a$ and $b \in C_{\Gamma}(a)$, then $b \cap x \in a$.

$\Gamma$-amenability is sometimes called $\Gamma$-completeness or $\Gamma$-fullness.

Definition 3.10 Let $N$ be countable and transitive; then $N$ is a coarse $\Gamma$ Woodin premouse iff

1. $N \models Z F C$,

2. $N$ is $\Gamma$-amenable, and 
3. letting $\delta=O R \cap N$, for any $f: \delta \rightarrow \delta$ such that $f \in C_{\Gamma}(N)$, there is a $\kappa<\delta$ such that $f^{\prime \prime} \kappa \subseteq \kappa$ and an $E$ such that $N \models E$ is an extender with critical point $\kappa$ and $\left.V_{i_{E}(f)(\kappa)} \subseteq U l t(V, E)\right)$.

Let $T$ be the tree of a $\Gamma$ scale on a universal $\Gamma$ set, where $\Gamma$ is good, and assume $A D$. Let $N$ be countable transitive and $\delta=O R \cap N$. It is easy to see then that $N$ is a coarse $\Gamma$-Woodin premouse iff $N=V_{\delta}^{L(N \cup\{T, N\})}$ and $L(N \cup\{T, N\}) \models \delta$ is Woodin.

Our condensation result 3.8 easily yields the following lemma.

Lemma 3.11 Assume $A D$, and let $\Gamma_{0}$ and $\Gamma_{1}$ be good pointclasses such that $\Gamma_{0} \subseteq \Delta_{1}$. Let $N$ be a coarse $\Gamma_{1}$ - Woodin premouse; then for some $\eta<$ $O R \cap N, V_{\eta}^{N}$ is a coarse $\Gamma_{0}$-Woodin premouse.

Proof. Let $T_{1}$ be the tree of a $\Gamma_{1}$ scale on a universal $\Gamma_{1}$ set, and let $T, U \in$ $L\left(N \cup\left\{T_{1}, N\right\}\right)$ be trees projecting to the universal $\Gamma_{0}$ set and its complement. Let $\delta=O R \cap N$, and let $M$ be a $\Sigma_{2}$ admissible set of the form $L_{\alpha}\left(N \cup\left\{T_{1}, N\right\}\right)$ such that $T, U \in M$. As $\delta$ is strongly inaccessible in $L\left(N \cup\left\{T_{1}, N\right\}\right)$ we can, working in this universe, form a hull of $M$ whose intersection with $V_{\delta}^{M}(=N)$ is of the form $V_{\eta}^{M}$ for some $\eta<\delta$. Letting $\bar{M}$ be the collapse of this hull, we have $C_{\Gamma_{0}}\left(V_{\eta}^{N}\right) \subseteq \bar{M}$ by 3.8. On the other hand, $\delta$ is Woodin in $M$, so $\eta$ is Woodin in $\bar{M}$. Therefore $V_{\eta}^{N}$ is a coarse $\Gamma_{0}$-Woodin premouse.

Finally, we can use the results of this section to construct a $\Gamma$-Woodin premouse having an iteration strategy which is reasonably close to $\Gamma$.

Lemma 3.12 Assume $A D$, and let $\Gamma_{0}, \Gamma_{1}$, and $\Gamma_{2}$ be good pointclasses such that $\Gamma_{0} \subseteq \Delta_{1}$ and $\Gamma_{1} \subseteq \Delta_{2}$; then for any real $y$ there is a coarse $\Gamma_{0}$-Woodin mouse $M$ such that $y \in M$, and $M$ has an $\omega_{1}$-iteration strategy $\Sigma$ such that

1. $\Sigma$ is coded by a set of reals in $\Gamma_{2}$, and

2. every iterate of $M$ via $\Sigma$ is a $\Gamma_{0}$-Woodin mouse.

Proof. Let $\Gamma_{3}$ and $\Gamma_{4}$ be further good pointclasses such that $\Gamma_{i} \subseteq \Delta_{i+1}$ for all $i<4$. It is a well known result of Woodin that, assuming $A D$, for any set $S$ of ordinals, $\operatorname{HOD}_{S}^{L[S, x]} \models\left(\omega_{2}^{L[S, x]}\right.$ is Woodin) for a cone of reals $x$. Applying this with $S=\left(T_{4}, y\right)$ we get a $\Gamma_{4^{-}}$Woodin premouse $P$ such that $y \in P$. 
Let $\eta<O R \cap P$ be least such that $V_{\eta}^{P}$ is a $\Gamma_{0^{-}}$-Woodin premouse, and notice that by 3.11 , for all $\xi \leq \eta, V_{\xi}^{P}$ is not $\Gamma_{1}$-Woodin, so that there is a function $f: \xi \rightarrow \xi$ in $C_{\Gamma_{1}}\left(V_{\xi}^{P}\right)$ witnessing non-Woodinness. Let $T$ and $U$ be trees for the universal $\Gamma_{1}$ set and its complement which are constructible from $T_{4}$. Let $N$ be a $\Sigma_{2}$ admissible set of the form $L_{\alpha}\left(V_{\eta}^{P} \cup\{T, U\}\right)$, and let $\pi: \bar{N} \rightarrow N$ be elementary, with $\pi, \bar{N}$ in $P$ and countable there, and $\pi(\bar{\eta}, \bar{T}, \bar{U})=(\eta, T, U)$.

Claim In $P$ it is true that $\bar{N}$ is $\omega_{1}$-iterable, for iteration trees formed using extenders from $V_{\bar{\eta}}^{\bar{N}}$ and its images via the strategy of choosing the unique $\pi$-realizable branch.

Proof. Work in $P$. From [3] we know that there is always a maximal such branch, so it suffices to see that if $\mathcal{T}$ on $\bar{N}$ has cofinal $\pi$-realizable branches $b$ and $c$, then $b=c$. Let $\delta=\delta(\mathcal{T})$ and $Q=V_{\delta}^{\mathcal{M}_{b}^{\mathcal{T}}}=V_{\delta}^{\mathcal{M}_{c}^{\mathcal{T}}}$. Let $\sigma$ and $\tau$ be the realizing maps for $b$ and $c$ respectively. Since $T$ and $U$ are in $\operatorname{ran}(\sigma), 3.8$ gives that $C_{\Gamma_{1}}(Q) \in \mathcal{M}_{b}^{\mathcal{T}}$ and $\sigma\left(C_{\Gamma_{1}}(Q)=C_{\Gamma_{1}}\left(V_{\xi}^{N}\right)\right.$, where $\xi=\sigma(\delta) \leq \eta$. By our choice of $\eta$, this implies there is an $f \in C_{\Gamma_{1}}(Q)$ witnessing non-Woodinness for $Q$. Since $C_{\Gamma_{1}}(Q) \in \mathcal{M}_{c}^{\mathcal{T}}$ by the same proof, $f \in \mathcal{M}_{b}^{\mathcal{T}} \cap \mathcal{M}_{c}^{\mathcal{T}}$. From [3], we get then $b=c$.

The proof of the claim easily gives

Claim In $P$, if $b$ is a cofinal $\pi$-realizable branch of a countable $\mathcal{T}$ on $\bar{N}$

using extenders from $V_{\bar{\eta}}^{\bar{N}}$ and its images, then setting $Q=V_{\delta(\mathcal{T})}^{\mathcal{M}_{b}^{\mathcal{T}}}, b$ is the unique cofinal branch $d$ of $\mathcal{T}$ such that $C_{\Gamma_{1}}(Q) \subseteq \mathcal{M}_{d}^{\mathcal{T}}$.

It follows easily from the claims that $P$ satisfies that $\bar{N}$ has a $\Gamma_{2}$ iteration strategy. But $P$ is $\Gamma_{4}$-correct, so this is indeed true.

\section{Proof of Theorem 1.5}

Our plan is to first prove that a version of the theorem holds on a cone ( that is, that certain inner model operators are fine structural), and then add the base of such a cone to a "lightface" mouse via Woodin's "every real generic" extender algebra. The next lemma executes the first step of the plan. 
Lemma 4.1 Assume $A D$, and let $\alpha<\theta$ be such that $P_{\alpha}(\mathbb{R})$ is closed under $\exists^{\mathbb{R}}$, every set in $P_{\alpha}(\mathbb{R})$ has a scale in $P_{\alpha}(\mathbb{R})$, and every premouse having an $\omega_{1}$-iteration strategy in $P_{\alpha}(\mathbb{R})$ is tame. Let $\Gamma \subsetneq P_{\alpha}(\mathbb{R})$ be good; then the inner model operator $x \mapsto C_{\Gamma}(x)$ is fine structural.

Proof. By 2.2, it is enough to find a fine structural inner model operator which is above $x \mapsto C_{\Gamma}(x)$ in $\leq_{m}$. By the comparability of inner model operators, this follows if we show that for any real $y$, there is an $x \geq_{T} y$, an $\omega_{1}$-iterable $x$-mouse $\mathcal{R}$, and a real $z \in \mathcal{R}$ such that $z \notin C_{\Gamma}(x)$. So fix a real $y$.

Let $\Gamma_{0}$ be a good pointclass such that $\Gamma \subseteq \Delta_{0} \subsetneq P_{\alpha}(\mathbf{R})$. Let $M$ be a coarse $\Gamma_{0}$-Woodin premouse which has an $\omega_{1}$-iteration strategy in $P_{\alpha}(\mathbb{R})$ and is such that $y \in M$; the existence of $M$ is guaranteed by 3.12. Let $\Omega=O R \cap M$, and $\left\langle\mathcal{N}_{\eta} \mid \eta \leq \Omega\right\rangle$ be the levels of the $L[\vec{E}, y]$ construction of [4] done inside $M$. (So $y$ is thrown in at the bottom, and we use full background extenders.) Since $M$ is fully iterable, all $\mathcal{N}_{\eta}$ are fully iterable, and the construction never breaks down.(Cf. [4].) As $\Omega$ is Woodin in $L(M \cup\{M\}), \Omega$ is Woodin in $\mathcal{Q}$, where $\mathcal{Q}$ is the premouse of height $O R$ whose $\Omega^{\text {th }}$ level $\mathcal{J}_{\Omega}^{\mathcal{Q}}$ is $\mathcal{N}_{\Omega}$. Since $\mathcal{Q}$ has an $\omega_{1}$-iteration strategy in $P_{\alpha}(\mathbf{R}), \mathcal{Q}$ is tame. It follows that for all sufficiently large $\eta<\Omega, \eta$ is not Woodin in $\mathcal{Q}$.

There is a club $B \subseteq \Omega$ in $L\left(M \cup T_{0}, M\right)$ such that for all $\eta \in B, V_{\eta}^{M}$ is $\Gamma$-Woodin and $\mathcal{N}_{\eta}=J_{\eta}^{\mathcal{Q}}$. (Here $T_{0}$ is the tree of a $\Gamma_{0}$ scale on a universal $\Gamma_{0}$ set. We actually use here the proof of 3.11, and not just its statement.) Fix $\eta \in B$ such that $\eta$ is not Woodin in $\mathcal{Q}$. Now $J_{\eta}^{\mathcal{Q}}$ is the output of the $L[\vec{E}, y]$-construction done up to $\eta$ in $L\left(V_{\eta}^{M} \cup\{T\}\right)$, where $T$ is the tree of a scale for $\Gamma$, and $\eta$ is Woodin in this universe. It follows from [4] that if $f: \eta \rightarrow \eta$ is in $C_{\Gamma}\left(V_{\eta}^{M}\right)$ and amenable to $J_{\eta}^{\mathcal{Q}}$, then $J_{\eta}^{\mathcal{Q}}$ is Woodin with respect to $f$. Since $\eta$ is not Woodin in $\mathcal{Q}$, we can fix a subset $b$ of $\eta$ which is in $\mathcal{Q}$ but not in $C_{\Gamma}\left(V_{\eta}^{M}\right)$. Let $\mathcal{P}=J_{\xi+1}^{\mathcal{Q}}$, where $b \in\left(J_{\xi+1}^{\mathcal{Q}} \backslash J_{\xi}^{\mathcal{Q}}\right)$.

Now let $g: \omega \rightarrow J_{\eta}^{\mathcal{Q}}$ be $Q$-generic for $\operatorname{Col}\left(\omega, J_{\eta}^{\mathcal{Q}}\right)$ and such that, setting $x=x_{g}$, we have $b_{x} \notin C_{\Gamma}(x)$; there are in fact comeager many such $g$. Clearly, $y \leq_{T} x$ and $b_{x} \in \mathcal{P}[x]$. It remains only to show that $\mathcal{P}[x]$ can be re-arranged as an $x$-mouse $\mathcal{R}$. This is a simple result due to the author. We define $\mathcal{R}$ by adding $E$ to the $\mathcal{R}$-sequence with index $\alpha$ just in case $\eta<\alpha, \alpha$ indexes an extender $F$ on the $\mathcal{P}$-sequence, and $E$ is the canonical extension of $F$ to $J_{\alpha}^{\mathcal{R}}=J_{\alpha}^{\mathcal{P}}[x]$ determined by the fact that that this structure is a small forcing extension of $J_{\alpha}^{\mathcal{P}}$. One can prove by induction on $\beta$, using the quantifier-by- 
quantifier definability of the forcing relation over $J_{\eta+\beta}^{\mathcal{P}}$, that $J_{\beta}^{\mathcal{R}}$ has the same projecta and standard parameters as $J_{\eta+\beta}^{\mathcal{P}}$, and hence is $\omega$-sound. We leave the details to the reader.

Proof of Theorem 1.5. For $B \subseteq \mathbb{R}$, let

$$
\mathcal{A}_{B}=\left(V_{\omega+1} \cup\left\{C^{*} \mid C \leq_{W} B\right\}, \in\right),
$$

where $C^{*}$ is the subset of $\mathrm{HC}$ coded by $C$. We wish to show that there is an $\omega_{1}$-iterable, fine structural premouse "over $\emptyset$ " whose reals are precisely those which are definable from a countable ordinal parameter over some $\mathcal{A}_{B}$ for $B \in P_{\alpha}(\mathbb{R})$. ( Here $P_{\alpha}(\mathbb{R})$ is as in the hypothesis of 1.5.)

For this, it will suffice to show that if $z$ is a real which is definable over $\mathcal{A}_{B}$ from a countable ordinal, and $B \in P_{\alpha}(\mathbb{R})$, then there is a (fine structural, lightface) premouse $\mathcal{Q}$ such that $z \in \mathcal{Q}$ and $\mathcal{Q}$ has an $\omega_{1}$-iteration strategy which is in $P_{\alpha}(\mathbb{R})$.(Proof: We may as well choose $\mathcal{Q}=\mathcal{Q}_{z}$ so that it projects to $\omega$ and is $\omega$-sound. Letting $\eta=O R \cap \mathcal{Q}$, we then have that $\mathcal{Q}$ is definable over $\mathcal{A}_{B}$ from $\eta$ as the unique $\omega_{1}$-iterable, $\omega$-sound premouse of height $\eta$ projecting to $\omega$, whenever $B$ is so large that $\mathcal{Q}$ has an iteration strategy in $\mathcal{A}_{B}$. If we then let $\mathcal{M}$ be the premouse whose proper initial segments are precisely the $\mathcal{Q}_{z}$ 's, $z \in \mathbb{R} \cap \mathcal{M}$ iff $z$ is definable from a countable ordinal over some $\mathcal{A}_{B}$ for $B \in P_{\alpha}(\mathbb{R})$.) So fix $z \in \mathbb{R}$ and $B \in P_{\alpha}(\mathbb{R})$ such that $z$ is definable over $\mathcal{A}_{B}$ from a countable ordinal, say by a $\Sigma_{n}$ formula.

Let $\Gamma \subsetneq P_{\alpha}(\mathbb{R})$ be a good pointclass such that $B \in \Delta$ and for all reals $x,\left(C_{\Gamma}\left(V_{\omega} \cup\{x\}\right), \in, B \cap C_{\Gamma}\left(V_{\omega} \cup\{x\}\right)\right)$ is a $\Sigma_{n+9}^{1}$-elementary substructure of $\left(V_{\omega+1}, \in, B\right)$. The closure of $P_{\alpha}(\mathbb{R})$ under $\exists^{\mathbb{R}}$ and scales enables us to find such a $\Gamma$. Let $\Gamma_{i}$, for $i \in \omega$, be a strictly increasing sequence of good pointclasses contained in $P_{\alpha}(\mathbb{R})$, with $\Gamma \subsetneq \Gamma_{0}$. By 4.1 we can fix a real $y$ such that for all $x \geq_{T} y, C_{\Gamma_{i}}(x)$ is a mouse set for all $i$. The proof of 4.1 also shows that if $w \in C_{\Gamma_{i}}(x)$, for $x \geq_{T} y$, then there is a $x-$ mouse $\mathcal{M}$ such that $w \in \mathcal{M}$ and $\mathcal{M}$ has an $\omega_{1}$-iteration strategy in $\Gamma_{i+1}$. (Cf. 3.12.) We may assume that $z \leq_{T} y$.

Claim 1. For any $x$ such that $y \leq_{T} x$, there is a fine-structural, $\omega_{1}+1$-iterable $x$-mouse $\mathcal{Q}_{x}$ such that

(a) $\mathcal{Q}_{x} \in C_{\Gamma}(x)$, 
(b) $\mathcal{Q}_{x}=$ ZFC + "there is a Woodin cardinal $\eta$ and an $\eta^{+}$-universally Baire set $B$ such that $z$ is ordinal definable over $\mathcal{A}_{B}$ ", and

(c) $\mathcal{Q}_{x}$ is pointwise definable (in the language of $x$-premice).

Proof. We argue just as in the proof of 4.1. Let $N$ be a coarse $\Gamma_{9}$-Woodin mouse having an $\omega_{1}$-iteration strategy in $P_{\alpha}(\mathbb{R})$ and such that $x \in N$. Let $\left\langle\mathcal{N}_{\eta} \mid \eta \leq \Omega\right\rangle$ be the models of the $L[\vec{E}]$ construction of $N$ done over the real $x$ ) of $N$. Just as in the proof of 4.1, we can fix an $\eta<\Omega$ such that $V_{\eta}^{M}$ is $\Gamma_{8}$-Woodin and $J_{\eta}^{\mathcal{N}_{\Omega}}=\mathcal{N}_{\eta}$, and such that some level of $\mathcal{N}_{\Omega}$ projecting to $\eta$ is not in $C_{\Gamma_{8}}\left(V_{\eta}^{N}\right)$. Let us choose $\mathcal{S}$ to be the first level $\mathcal{P}$ of $\mathcal{N}_{\Omega}$ such that $\mathcal{P}$ projects to $\eta$ and $\mathcal{P} \notin C_{\Gamma_{8}}\left(\mathcal{N}_{\eta} \cup\left\{\mathcal{N}_{\eta}\right\}\right)$. Notice that $\mathcal{S} \models(\eta$ is Woodin $)$.

By our choice of $\mathcal{S}$, there are comeager many $f: \omega \rightarrow J_{\eta}^{\mathcal{S}}$ such that $\mathcal{S}$ is not coded by any real in $C_{\Gamma_{8}}\left(x_{f}\right)$. We can therefore fix such an $f$ which is $\operatorname{Col}\left(\omega, J_{\eta}^{\mathcal{S}}\right)$ generic over $\mathcal{S}$. Let $w=x_{f}$. Clearly, $x \leq_{T} w$. Also, $w$ is $\mathcal{S}$ generic over a poset of size $\eta$ in $\mathcal{S}$, and $w$ codes $J_{\eta}^{\mathcal{S}}$, so by the level-by-level definability of forcing we can find a $w$-premouse $\mathcal{R}$ whose universe is $\mathcal{S}[w]$. The iterability of $\mathcal{S}$ guarantees that of $\mathcal{R}$. Since $\mathcal{S}$ projects to $\eta, \mathcal{R}$ projects to $\omega$. By our choice of $w$, the real canonically coding $\mathcal{R}$, its first order theory with parameter $w$, is not in $C_{\Gamma_{8}}(w)$. On the other hand, every proper initial segment of $\mathcal{S}$ projecting to $\eta$ is in $C_{\Gamma_{8}}\left(J_{\eta}^{\mathcal{S}}\right)$, and therefore every proper initial segment of $\mathcal{R}$ projecting to $\omega$ is in $C_{\Gamma_{8}}(w)$. But $w$ is in the cone above $y$, so that $C_{\Gamma_{8}(w)}$ is a mouse set. By comparison we see easily that in fact $\mathbb{R} \cap \mathcal{R}=C_{\Gamma_{8}}(w)$.

Subclaim A. There is a $\gamma<\left(\eta^{+}\right)^{\mathcal{S}}=\omega_{1}^{\mathcal{S}[w]}$ such that

(a) $\mathcal{S}|\gamma|=$ every set has cardinality $\eta$,

(b) $\mathbb{R} \cap \mathcal{S}[w] \mid \gamma$ is $C_{\Gamma_{3}}$-closed, and

(c) there is no $C_{\Gamma_{3}}(\mathcal{S}[w] \mid \gamma)$ collapsing structure for $\mathcal{S}[w] \mid \gamma$, in the sense that if $\mathcal{S}[w] \mid \xi \in C_{\Gamma_{3}}(S[w] \mid \eta)$, then $\gamma$ is still uncountable in $\mathcal{S}[w] \mid \xi$.)

Proof sketch. If subclaim A fails, we could show $C_{\Gamma_{8}}(w) \subseteq C_{\Gamma_{7}}(w)$, as we'd have for $w$-premice $\mathcal{M}$ projecting to $\omega$

$$
\mathcal{M}=\mathcal{S}[w] \mid \xi \text { for some } \xi<\omega_{1}^{\mathcal{S}[w]}
$$


if and only if

$\forall \alpha\left(\mathcal{M} \mid \alpha\right.$ is iterable above its $\omega_{1}$ via a $\Gamma_{4}$ iteration strategy. $)$

We use here that for $y \leq_{T} s, C_{\Gamma_{3}}(s)$ has been captured by mice with $\Gamma_{4}$ iteration strategies.

Fix $\gamma$ as in subclaim A. Since $\gamma$ is countable in $\mathcal{S}[w]$, we can fix $\xi$ least such that $\gamma$ is countable in $\mathcal{S}[w] \mid(\xi+1)$.

Subclaim B. $\gamma=\eta^{+}$in $\mathcal{S}|\xi, \mathcal{S}| \xi$ projects to $\eta$, and $C_{\Gamma}(\mathcal{S} \mid \gamma) \subseteq \mathcal{S} \mid \xi$.

Proof. The first two assertions are immediate consequences of the corresponding facts for $\mathcal{S}[w]$, using the forcing relation. For the third, fix $X \in C_{\Gamma}(\mathcal{S} \mid \gamma$.

Note that $(\mathcal{S} \mid \xi)[w]$ projects to $\omega$, and so is coded in a simple way by a real $u$ such that $u \notin C_{\Gamma}((\mathcal{S} \mid \gamma)[w])$. It follows that $\mathcal{S} \mid \xi$ projects to $\eta$, and if $Y \subseteq \eta$ codes $\mathcal{S} \mid \xi$ via its theory in the natural way, then $Y \notin C_{\Gamma}(\mathcal{S} \mid \gamma)$.

But then for comeager many $u: \omega \rightarrow \mathcal{S} \mid \eta$, the theory of $(\mathcal{S} \mid \xi)[u]$ is not in $C_{\Gamma}((\mathcal{S} \mid \gamma)[u]$. Now any such $u$ is essentially a real in the cone above $y$, and $\mathcal{S}[u]$ is a $u$-mouse. By our choice of $y$, and a comparison argument, we have that $C_{\Gamma}((\mathcal{S} \mid \gamma)[u]) \subseteq(\mathcal{S} \mid \xi)[u]$, and hence that $X \in(\mathcal{S} \mid \xi)[u]$. Since this last statement is true for comeager many $u$, we have that $X \in \mathcal{S} \mid \xi$, as desired.

We can now finish the proof of claim 1. Let $T$ be the tree of a scale on a universal $\Gamma$ set. Since $B \in \Delta$, there are trees $U_{0}, U_{1}$ in $L[T]$ such that $p\left[U_{0}\right]=B$ and $p\left[U_{1}\right]=\neg B$. By a Skolem hull argument conducted in $L[T, \mathcal{S} \mid \gamma]$, we can find trees $\bar{U}_{0}, \bar{U}_{1}$ in $L[T, \mathcal{S} \mid \gamma]$, and having cardinality $\gamma=\eta^{+}$ in $L[T, \mathcal{S} \mid \gamma]$, such that whenever $g$ is $\mathcal{S}$-generic over $\operatorname{Col}(\omega, \eta)$, then

$$
p\left[\bar{U}_{0}\right] \cap(\mathcal{S} \mid \gamma)[g]=B \cap(\mathcal{S} \mid \gamma)[g],
$$

and

$$
p\left[\bar{U}_{1}\right] \cap(\mathcal{S} \mid \gamma)[g]=\neg B \cap(\mathcal{S} \mid \gamma)[g] .
$$

By the result of Harrington and Kechris, $\bar{U}_{0}$ and $\bar{U}_{1}$ are in $C_{\Gamma}(\mathcal{S} \mid \gamma)$, and hence by subclaim B, they are in $\mathcal{S} \mid \xi$. Since $\gamma=\eta^{+}$in $\mathcal{S} \mid \xi$, we see from the formulae just displayed that

$$
\mathcal{S} \mid \xi \models \bar{U}_{0}, \bar{U}_{1} \text { witness that } B \cap \mathcal{S} \mid \xi \text { is } \eta^{+} \text {-universally Baire. }
$$


(Note that $B \cap \mathcal{S}|\xi \in \mathcal{S}| \xi$ by subclaim B.)

We leave it to the reader to show that there is a $\xi^{*}$ such that $\gamma<\xi^{*} \leq \xi$, $\bar{U}_{0}, \bar{U}_{1} \in \mathcal{S} \mid \xi^{*}$, and $\mathcal{S} \mid \xi^{*} \models$ ZFC. Clearly, $\mathcal{S} \mid \xi^{*}$ satisfies demands (a) and (b) on $\mathcal{Q}_{x}$ of Claim 1. (We use the fact that $V_{\omega+1} \cap \mathcal{S} \mid \xi^{*}$ is $C_{\Gamma^{-}}$-closed to show that $\mathcal{S} \mid \xi^{*}$ satisfies that $z$ is OD over $\mathcal{A}_{B \cap \mathcal{S} \mid \xi^{*}}$.) In order to satisfy demand (c) as well, we simply take $\mathcal{Q}_{x}$ to be the pointwise-definable (in the language of $x$-premice) Skolem hull of $\mathcal{S} \mid \xi^{*}$. This proves claim 1.

Claim 2. Let $y \leq_{T} x$, and let $\mathcal{Q}_{x}$ be as in claim 1. Then $z$ is ordinal definable over $\mathcal{Q}_{x}$.

Proof. Working in $\mathcal{Q}_{x}$, and letting $B$ be the witness to part (b) of claim 1 , we have that $B$ is $\eta^{+}$-universally Baire, where $\eta$ is a Woodin cardinal. Thus $B$ is $<\eta$-homogeneous, so that the Wadge hierarchy below $B$ is semiprewellordered. It follows that $\mathcal{A}_{B}$ is definable from the Wadge ordinal of $B$, and thus $z$ is ordinal definable.

We are ready to complete the proof of 1.5 . Recall that we are seeking a "lightface" premouse $\mathcal{Q}$ such that $z \in \mathcal{Q}$, and $\mathcal{Q}$ has an $\omega_{1}$-iteration strategy in $P_{\alpha}(\mathbb{R})$. We get $\mathcal{Q}$ by running yet again the proof of 4.1.

So let $M$ be an $\omega_{1}$-iterable, coarse $\Gamma_{9}$-Woodin mouse such that $y \in M$. Let $L[\vec{E}]$ be the output of the fully backgrounded "lightface" $K^{c}$-construction done inside $M$. As before, since $L[\vec{E}]$ does not reach a Woodin limit of Woodins, we can fix an $\eta<\mathrm{OR}^{M}$ such that $V_{\eta}^{M}$ is $\Gamma_{8}$-Woodin, but $L[\vec{E}] \models \eta$ is not Woodin. Let $\mathcal{Q}$ be the corresponding $\mathcal{Q}$-structure, that is, $\mathcal{Q}=\mathcal{J}_{\gamma}^{\vec{E}}$, where $\gamma$ is least such that some $f: \eta \rightarrow \eta$ witnessing non-Woodinness of $\mathcal{J}_{\eta}^{\vec{E}}$ is in $\mathcal{J}_{\gamma+1}^{\vec{E}}$. Thus (the subset of $\eta$ naturally coding) $\mathcal{Q}$ is not in $C_{\Gamma_{8}}\left(V_{\eta}^{N}\right)$. Since all levels of $L[\vec{E}]$ are tame, $\eta$ is a cutpoint of $\mathcal{Q}$. Clearly, $\mathcal{Q}$ has an $\omega_{1}$-iteration strategy in $P_{\alpha}(\mathbb{R})$. We shall show $z \in \mathcal{Q}$.

Let $\mathbb{P}$ be the every-real-generic poset of $\mathcal{Q}$ (up to $\eta$ ). Here we only use extenders from the $J_{\eta}^{\mathcal{Q}}$ sequence which are total and strong out to their lengths to define the identities. Since the $J_{\eta}^{\mathcal{Q}}$ sequence has background extenders from $V_{\eta}^{M}$ (which haven't been collapsed in the construction) for these extenders on the $J_{\eta}^{\mathcal{Q}}$ sequence, every real in $M$ is $\mathbb{P}$ generic over $\mathcal{Q}$. In particular, $y$ is so generic.

By our choice of $\mathcal{Q}$, there are comeager many $f: \omega \rightarrow J_{\eta}^{\mathcal{Q}} \cup\{y\}$ such that $\mathcal{Q}$ is not coded by any real in $C_{\Gamma_{8}}\left(x_{f}\right)$. We can therefore fix such an $f$ which 
is $\operatorname{Col}\left(\omega, J_{\eta}^{\mathcal{Q}} \cup\{y\}\right)$ generic over $\mathcal{Q}[y]$. Let $x=x_{f}$. Clearly, $y \leq_{T} x$. Also, $x$ is $\mathcal{Q}$ generic over a poset of size $\eta$ in $\mathcal{Q}$, and $x$ codes $J_{\eta}^{\mathcal{Q}}$, so by the level-by-level definability of forcing we can find an $x$-premouse $\mathcal{R}$ whose universe is $\mathcal{Q}[x]$. The iterability of $\mathcal{Q}$ guarantees that of $\mathcal{R}$. Since $\mathcal{Q}$ projects to $\eta, \mathcal{R}$ projects to $\omega$. By our choice of $x$, the real canonically coding $\mathcal{R}$, its first order theory with parameter $x$, is not in $C_{\Gamma_{8}}(x)$. Since $x$ is in the cone above $y$, we have by comparison that $C_{\Gamma_{8}}(x) \subseteq \mathcal{R}$.

Letting $\mathcal{Q}_{x}$ be as in claim 1 , we have by a comparison argument that $\mathcal{Q}_{x}=\mathcal{R} \mid \xi$ for some $\xi$. That is,

$$
\mathcal{Q}_{x}=(\mathcal{Q} \mid \xi)[x]
$$

where the two are considered only as structures for the language of set theory. But then $z$ is ordinal definable over $(\mathcal{Q} \mid \xi)[x]$, which is a homogeneous forcing extension of $\mathcal{Q} \mid \xi$. Thus $z \in \mathcal{Q} \mid \xi$, as desired.

This completes the proof of 1.5

\section{References}

[1] L. Harrington and A.S. Kechris, On the determinacy of games on ordinals, Annals of Math. Logic, vol. 20 (1981), 109-154.

[2] A.S. Kechris, The theory of countable analytical sets, Transactions of the AMS, 202 (1975), 259-297.

[3] D.A. Martin and J. R. Steel, Iteration trees, Journal of the AMS, vol. 7 (1994) 1-73.

[4] W.J. Mitchell and J. R. Steel, Fine structure and iteration trees, Springer Lecture Notes in Logic vol. 3, Springer-Verlag, 1994.

[5] M. Rudominer, Mouse sets definable in L(R), Ph.D. thesis, UCLA, 1994.

[6] J.R. Steel, A classification of jump operators, Journal of Symb. Logic vol. 47 (1982) 347-358.

[7] J.R. Steel, Inner models with many Woodin cardinals, Annals of Pure and Applied Logic vol. 65 (1993) 185-209. 
[8] J.R. Steel, Projectively wellordered inner models, Annals of Pure and Applied Logic, vol.74 (1995) 77-104.

[9] J.R. Steel, Woodin's analysis of $\operatorname{HOD}^{L(\mathbb{R})}$, available at http://math. berkeley.edu/ steel 